\title{
An Efficient and Effective Wavelet Transform Methods for OCT, SD-OCT and Secondary Image Compression
}

\author{
Dr.V.Kathiresan \\ HOD, Department of Computer Applications \\ Dr.SNS Rajalakshmi College of Arts and Science \\ Coimbatore-49,India
}

\author{
S.Nithya \\ Research Scholar \\ Department of Computer Science, \\ Dr.SNS Rajalakshmi College of Arts and Science \\ Coimbatore-49,India
}

\begin{abstract}
Glaucoma is a chronic eye disease that leads to vision loss Glaucoma is caused due to the increase in intraocular pressure of the eye. The intraocular pressure increases due to malfunction or malformation of the drainage system of the eye. A Dual Tree Complex Wavelet Transform (DTCWT) based image compression is proposed to solve factors such as low image contrast, speckle noise, high spatial resolution. DTCWT is used to compress the OCT, SD-OCT and secondary images and it is efficient for detecting glaucoma compared with the existing detection algorithms. Then the compression of OCT, SD-OCT and secondary images are carried out by 2D-oriented Biorthogonal Wavelet Transform (2D-BWT). In this BWT, effective coding performance is achieved by the Windowed All Phase Digital Filter (WAPDF) based on Discrete Cosine Transform (DCT). In BWT, Context-adaptive binary arithmetic coding (CABAC) with Lattice Vector Quantization (LVQ) is presented to encode the wavelet significant coefficients for finding the compressed image. The experimental result has demonstrated the significant improvement of the 2D-BWT with WAPDF gain for OCT, SD-OCT and secondary images with high resolution. Then apply Quincunx mexican-hat-like Wavelet Transform (QWT) with modified Support Vector Clustering (mSVC) proposed to evaluate the OCT, SD-OCT and secondary images. It is used to improve the image quality and reduced the image size for storage, an efficient image compression scheme has been presented by using transform and clustering schemes. The result proves that proposed QWT with mSVC system provides higher CR, correlation, PSNR, SSIM and lower MSE, execution time for OCT, SDOCT and secondary images significantly.
\end{abstract}

Key words: BWT, DCT, WAPDF, Glaucoma, OCT, SD-OCT, secondary images.

\section{INTRODUCTION}

Image compression is an application of data compression that encodes the original image with few bits. The objective of image compression is to reduce the redundancy of the image and to store or transmit data in an efficient form. Image processing usually refers to digital image processing, but optical and analog image processing also are possible [1]. Image processing is a rapidly growing area of computer science. Fields which traditionally used analog imaging are now switching to digital systems, for their flexibility and affordability. Important examples are medicine, film and video production, photography, remote sensing, and security monitoring [2].

Glaucoma is a disease of the retina which is one of the most common causes of permanent blindness worldwide. The disease causes damage to the retinal ganglion cells, resulting in thinning of the retinal nerve fiber layer (RNFL). There is a fluid called aqueous humor that flows through the pupil and is absorbed by the bloodstream [3]. In case of glaucoma the flow of this fluid becomes clogged. This results more intraocular pressure in the eye which damages the highly sensitive optic nerve causing impairment. It mainly affects the portion inside the optic disk where the size of the optic cup increases resulting in a high cup-to-disk ratio. It causes the successive narrowing of the field of view of affected patients.

Abnormalities in the RNFL on long standing optic tract lesions are also characteristic. In such cases, there is a "wedge" or "band" of horizontal RNFL loss and pallor of the optic disc in the eye contralateral to the lesion (the eye with the temporal field loss). This pattern is caused by atrophy of the RNFL originating nasal to the fovea and is identical with that from chiasmal syndromes identified on ophthalmoscope as band atrophy (BA) of the optic nerve [4]. At the same time, there is generalized pallor of the optic disc in the eye on the side of the lesion associated with severe loss of the RNFL in the superior and inferior arcuate lesions that comprise the majority of fibers originating from the ganglion cells temporal to the fovea. The RNFL loss may be evaluated on ophthalmoscope as well as with several instruments including scanning laser polarimetric and optical coherence tomography.

Optical Coherence Tomography (OCT) is a noninvasive measurement method that uses light with low temporal coherence to create two- and three dimensional tomograms of biological tissue in vivo such as the retina, skin, esophagus and coronary artery [5]. An OCT system is built around an interferometer, usually a Michelson interferometer, and consists of a source-, sample-, reference- and detector arm. In the source arm, a broadband source emits light with different wavelengths (low temporal coherence), preferably spatially confined. An optical isolator may be used to protect the source from back reflection. The source light enters the interferometer, where it is split between the sample and reference arms [6].

Spectral-domain Optical Coherence Tomography (SD-OCT) is a new, promising variant of OCT, providing superior sensitivity and measurement speed for cross- 
sectional imaging of turbid materials. OCT, originally developed for applications in the field of biomedical diagnostics, has shown high potential for other nonbiomedical investigation tasks, e.g. within the field of nondestructive testing (NDT) and contactless material characterization.

In wavelet based image coding, a variety of orthogonal and biorthogonal filters have been developed by researchers for signal analysis and compression. The selection of wavelet filters plays a crucial part in achieving an effective coding performance, because there is no filter that performs the best for all images [7]. The Current compression system uses the biorthogonal wavelet filters instead of orthogonal.

\section{MATERIALS AND METHODS}

\subsection{Dual Tree Complex Wavelet Transform Based Compression of Optical Coherence Tomography Images for Glaucoma Detection using Modular Neural Network}

In this research, preprocessing is performed on OCT images. Kuan filter is used to denoising and smoothen the image without removing edges or sharp features in the images. In this process, the multiplicative noise model transforms into a signal-dependent additive noise model. After that, the minimum Mean Square Error (MSE) criterion is applied to the model. It makes no approximation to the original form, it can be measured as better to the Lee filter.

Image enhancement technique is used to improve a given image quality. So that the result obtained from image enhancement is additional useful than the original image for RNFL boundaries detection. This method sharpens image features like edges, boundaries, or contrast for making an image with high quality for further processing.

Feature extraction is done by using Grey-Level Cooccurrence Matrix (GLCM). Seven properties from GLCM are computed, i.e., energy measuring uniformity of local grey scale distribution; correlation measuring the joint probability of occurrence; entropy measuring randomness; contrast measuring the local variations; cluster shade that measures a category of pixels having similar values of grey level; homogeneity that measures the closeness corresponding to the distribution and the inverse difference moment measuring local minimal variations. In this system, 28 features are extracted and also the average values of all angles utilized to obtain 7 features.

The feature selection is done by using Principal Component Analysis (PCA). The PCA is for the reason of minimizing the huge data dimensionality. In training phase, the feature vectors are extracted for each image present to produced training set. During the testing period, the feature vector equivalent to the test image and is computed employing PCA.

WWw.ijcat.com
Improved Artificial Bee Colony (IABC) clustering is used to discover boundaries of the RNFL. This algorithm grouping the glaucoma identical pixels region into one group and natural (normal) value of all pixels reward in that another group. The classification process is done by using Modular Neural Network (MNN). Every component in the collection associates with a MNN among the same structure and it simply defined as five experts with one gating network.

Image compression is performed by using DTCWT method. In this process, a gray scale OCT image is chosen to compress the image more effectively. The actual image undergoes decomposition by using DTCWT. The coefficients received are transformed by threshold for compression further applying arithmetic encoding in the process of entropy.

Wavelet transform has disadvantages like shift sensitivity, absence of directional selectivity and Absence of phase information, hence it does not find its application in several domains. DTCWT surpasses these above mentioned limitations. Two DWT's can generate separately the real and imaginary coefficients, only if, both of the filters design are particularly diverse from each other. Upper DWT generates the real part whereas the lower DWT yields the imaginary part. In case both are the same, then there is no gain. The realization consists of two steps. Initially, the decomposition of an input image is carried out by two branches ' $a$ ' and ' $b$ ' in which the upper DWT is the Hilbert transform (approximate) connected with the lower DWT [8]. Secondly, the same pass bands of the respective two sub bands are combined in a linear manner either through differencing or averaging.

In compression process, DTCWT is used over the input image that either brings the coefficients nearer or equivalent to zero. Additionally, the threshold $\boldsymbol{\lambda}$ also produces more zeros. $\lambda$ is fixed and the value which is below $\lambda$ is fixed to zero to produce more zeros in the hard thresholding, which, actually needs lesser space for storage and employing the entropy coding, the transmission tends to become more faster. Entropy coding is conducted by means of arithmetic coding for the purpose of compressing the image.

In this research work, arithmetic coding is used where the input symbol is substituted by a particular code in a file or message and this particular code is indicated by an integer number ranging between 0.0 and 1.0 of a real number. Short and long code words are allocated to more and less probable events correspondingly. This statistic tends to become accurate when the coding result attains the Shannon's entropy limit for the input symbols in a bigger sequence. Arithmetic coding comprises of three registers in the form of low, high and range.

2.2 A Biorthogonal Wavelet Transform Based Efficient Image Compression for SpectralDomain Optical Coherence Tomography Optimal Window Sequence of BWT with WAPDF for Image Compression by MCSO 
In this method, the first step chooses the SD-OCT source image by which the direction finder is enables finding of the transform direction. The decomposition of the image decomposition wavelet coefficients (w) is based on BWT with WAPDF. The thresholds help in employing the modified coefficients $w$. Later, is the wavelet significant coefficients is encoded based on CABAC with LVQ. The reconstruction of the image is done using the decoding and inverse of BWT with WAPDF.

In Modified Cat Swarm Optimization (MCSO), the adaptive parameters are considered. The method of optimization in MCSO avoids the use of any gradient information of the objective function and hence it operates on the simple concept making easy and efficient implementation while computing the algorithm. Therefore, MSCO enables to achieve the optimal window sequence of BWT with WAPDF for image compression. In this method, quantization phase is given as the "quality factor" $\mathrm{Q}$ as an important parameter in bit rate control.

The optimal Karhunen-Loeve transform (KLT) is given by the robust approximation given by the DCT. Given the assumption of the input signal as a first-order Gaussian-Markov process having zero-mean, unit variance and correlation coefficient $\rho=0.95$, a good approximation is fixed for natural images by which the coding gain of the DCT is in close proximity with that of the optimal mode of KLT [9]. Hence, the proposed method represents the good decorrelation ability.

The pixel sequences are quantized rather than just the vector quantizer (VQ) for single pixels is used. The derivation of the LQ codebook is given by selecting a finite number of lattice points from the finite lattice. However, the determination of LVQ is given by a truncation, a root lattice and a scaling factor in which the root lattice is defined as the lattice co set from which the actual building of codebook is performed. In root lattice, the finite number of lattice points are chose based on truncation and thereby the input data is quantized with respect to finite energy. In the truncated area, the selection of bit rate of the LVQ is based on the number of points. Hence, scaling is required to truncate the lattice properly forgetting the best accommodation to the source probability distribution. It is must to achieve the information about the number of required lattice points within the truncated area, which can also be stated as the shape awareness of the truncated area.

The method of lossless compression is given as the entropy coding which is further utilized at the last stage of encoding the wavelet coefficients for image reduction which is done to a series of syntax elements. By defining the means using the reconstruction of the image coefficients, the syntax elements are used at the decoder. This is also called as inclusive prediction method given by intra prediction mode in either spatial or temporal prediction and motion vectors and also prediction error are termed as residual. In CABAC, three different functions such as binarization, context modeling, and arithmetic coding are considered. In binarization, the mapping of the syntax elements are given to binary symbols (bins). In case of context modeling, the estimation of the probability of the bins is shown. Finally, the arithmetic coding compresses the bins to bits with respect to the estimated probability.

CABAC employs the Binary Arithmetic Coding in which the binary decisions are specified in terms of encoding in either1 or 0 . For example, a transform coefficient or motion vector is considered as a non-binaryvalued symbol which is later binarized or modified into a binary code well ahead of the arithmetic coding. This process helps in data conversion showing the data symbol into a variable length code inspite of encoding the binary code after which the arithmetic coder is processed for before transmission. There is a repetition is phases for every bit of the binarized symbol. The non-binary valued syntax element of binary representation given by the binarization process must be close to a least redundancy code. In contrast, the most probable symbols are given easier access through the binary decisions placed in closer to the root node for the next modeling stage. Similar to this process, a code tree enables the reduction of the number of binary symbols for encoding on the average, Hence minimization of the computational workload helps to bring about the binary arithmetic coding stage.

Context modeling represents the accurate estimation of the probability approximate requisite to achieve the fast coding efficiency. This consequently represents the higher adaptive several models to be utilized for various bins and the probability of that context model is simplified depending on the values of the earlier coded bins. Bins having the similar distributions frequently share the similar context model. Based on the type of syntax element, the context model for each bin can be chosen by which bin position in syntax element is given asluma/chroma, binIdx, and neighboring information and so on. The context switch can be attained after each bin.

\subsection{An Efficient Image Compression Scheme based on Quincunx Mexican-Hat- Like Wavelet Transform with Modified Support Vector Clustering for SD-OCT Image}

In this work, a novel image compression scheme has been presented by using QWT and the modified SVC technique. In QWT, the images are divided into textual significance regions to extract textually important characteristics and employing textural descriptors as criteria. Then, these are including co-occurrence matrices based measures. While glaucoma SD-OCT image compression methodologies utilizing the QWT and applying it to the whole original image, and then applied threshold scheme to involve a more sophisticated scheme. Specifically, different compression ratios are transfer to the wavelet coefficients based on the different Regions of Interest (ROI), in which either each and every wavelet domain band of the image is clustered itself.

In the proposed QWT-mSVC, initially the input image is selected from image database. Then the images are separated into textual regions. These regions are clustered by two classes like significant region and non-significant 
region by using mSVC approach. First, the SD-OCT images are scanned with $\mathrm{M} \times \mathrm{M}$ dimensions of sliding windows. Here, 256 x 256 size of images are considered and the dimension is $\mathrm{M}=8$, and it is denoted as size of sliding window. The texture identification analysis is performed by using co-occurrence matrices [10]. The entry of matrices is represented as $(m, n)$ and it denotes the probability is going from one pixel with grey level $(m)$ to another pixel with grey level (n) based on the predefined angle and distance. For specific spatial distance and angles, new matrices are formed.

It checks if cooccurrence matrices derived features are used when a pattern has been marked as texturally significant, after that, the upper-left point of the equivalent sliding window takes on the label of 255 , else the label of zero has been taken. Based on this condition, new black-white image (i.e IMP) results show the significant region and non-significant region for each original gray level image. Then QWT compression has been introduced. In this compression, first the original image is decomposed into two images like $O I_{1}$ and $O I_{2}$. The wavelet representation is $Q W T-O I_{1}$ and $Q W T-\mathrm{OI}_{2}$ and their compression ratio is determined by using quantization procedure. The quantization preferred bit discrete stage for performance compared to common style of applying quantization. After quantization, the results are transferred to run length coding [11]. It is mainly used for reduced the redundancy problem in large data compression.

In Modified Support Vector Clustering (MSVC), handles the arbitrary shape clusters effectively. In discrete dyadic wavelet transform (DyWT) analysis, three families of wavelet are required and it considered as a drawback. This problem has been solved by two successive scales but the cost and loss of filter caused the computation complexity. So to solve these problems, quincunx has been introduced in [12]. In this system, follow this idea the wavelet compression has been done. In this decomposition, onlu fewer subbands only there compared than other decompositions, and that features are may produce lower visual quality of reconstructed image. But, this problem also overcomes in [13]. The simulations show that the quincunx decomposition performs efficiently in many practical applications.
In vector quantization, the Quantizer is used to reduce the number of bits required to store the transformed coefficients through decreasing the precision of those values. It is mainly used for image compression, because it has theoretical benefit over the scalar quantization schemes. Generally, during the encoding phase, the codebook design and the vector lookup is computationally complex. So, the codebook has been generated before encoding process as well as the efficiency of lookup table is more significant. In this work, the encoding values are collected from a multidimensional vector space into a finite set of values, which is collected from a discrete subspace of lower dimension. In addition, the lower space vector needed less storage space, therefore the image compression has been easily compressed.

In run length encoding, when the probability of frequency of the corresponding symbol is higher entropy coding substitutes a series of codes for a sequence of symbols, where the codes are chosen to have fewer bits. In this process, the redundancy has been reduced in the form of repeated bit patterns in the output of the Vector Quantizer (VQ). The RLE scheme only encodes the successive number of same color pixels the probability of occurrence of consecutive same color is very high. It provides competent compression of data, while the data with large number of runs or large number pixel contains same intensity value. Finally, the compressed image is reconstructed. It is efficiently decompress the image. The important and non-important textual region clustering has been discussed in given below subsection. It considers a secondary image which is evaluated by using the QWT with mSVC algorithm.

\section{RESULT AND DISCUSSION}

The Table 1 and 2 describes that the OCT, SD-OCT and secondary images are implemented by using proposed QWT-mSVC, 2D- BWT with WAPDF, 2D-DTCWT and existing DWT methods efficiently. From the Table 1 and Table 2 observes that the proposed QWT-mSVC method provides better performance metrics than other methods. Compare than OCT image, the SD-OCT and secondary images are obtained higher PSNR, CR, correlation, SSIM and lower MSE, execution time values.

Table 1: DWT and 2D-DTCWT methods for OCT, SD-OCT, secondary images

\begin{tabular}{|l|l|l|l|l|l|l|l|l|l|l|l|l|}
\hline \multicolumn{9}{|c|}{} & \multicolumn{1}{|c|}{ DWT-DTCWT } \\
\hline IMAGES & CR & PSNR & MSE & CORRELATION & SSIM & $\begin{array}{l}\text { EXECUTION } \\
\text { TIME }\end{array}$ & CR & PSNR & MSE & CORRELATION & SSIM & $\begin{array}{l}\text { EXECUTION } \\
\text { TIME }\end{array}$ \\
\hline OCT & 1.0073 & 15.9498 & 53.0413 & 0.8412 & 0.6147 & 0.3483 & 1.1985 & 27.5938 & 15.7144 & 0.9377 & 0.8899 & 0.1281 \\
\hline SD-OCT & 1.0492 & 21.4435 & 29.1048 & 0.8761 & 0.7269 & 0.3112 & 1.1999 & 28.7187 & 14.2719 & 0.9399 & 0.8923 & 0.1172 \\
\hline $\begin{array}{l}\text { Secondary } \\
\text { image }\end{array}$ & 1.0361 & 22.9114 & 29.4476 & 0.8832 & 0.7172 & 0.2942 & 1.1966 & 26.2995 & 13.4099 & 0.9456 & 0.8341 & 0.1191 \\
\hline
\end{tabular}

www.ijcat.com 
Table 2: 2D-BWT with WAPDF and QWT- mSVC methods for OCT, SD-OCT, secondary images

\begin{tabular}{|c|c|c|c|c|c|c|c|c|c|c|c|c|}
\hline \multicolumn{7}{|c|}{ QWT with 2D- BWT with WAPDF } & \multicolumn{6}{|c|}{ QWT-mSVC } \\
\hline IMAGES & CR & PSNR & MSE & CORRELATION & SSIM & $\begin{array}{l}\text { EXECUTION } \\
\text { TIME }\end{array}$ & CR & PSNR & MSE & CORRELATION & SSIM & $\begin{array}{l}\text { EXECUTION } \\
\text { TIME }\end{array}$ \\
\hline $\mathrm{OCT}$ & 1.2256 & 27.4921 & 13.3318 & 0.9582 & 0.9232 & 0.1214 & 1.3512 & 31.9894 & 11.9106 & 0.9670 & 0.9624 & 0.1017 \\
\hline SD-OCT & 1.2363 & 29.7381 & 12.2205 & 0.9476 & 0.9643 & 0.1159 & 1.4978 & 37.9004 & 10.1998 & 0.9876 & 0.9796 & 0.1011 \\
\hline $\begin{array}{l}\text { Secondary } \\
\text { image }\end{array}$ & 1.2565 & 31.4687 & 11.7895 & 0.9594 & 0.9600 & 0.1145 & 1.4491 & 38.9594 & 10.0006 & 0.9699 & 0.9674 & 0.100 \\
\hline
\end{tabular}

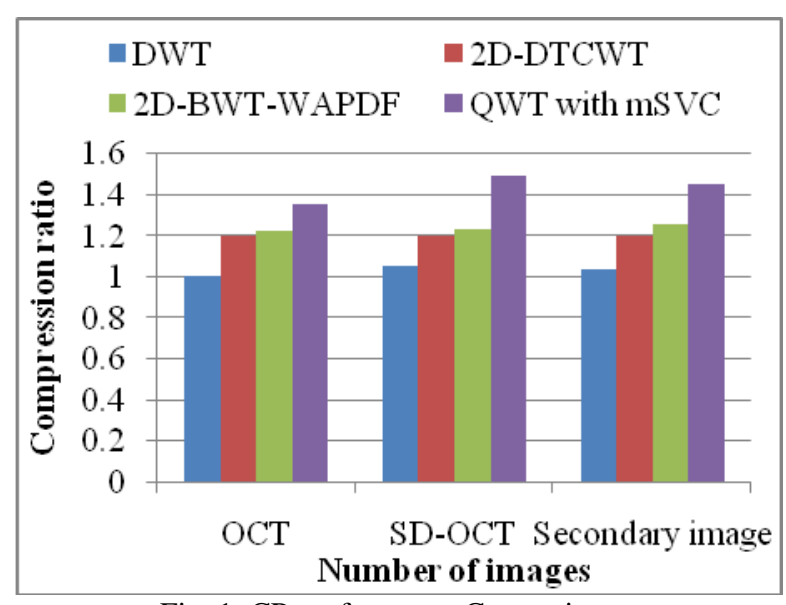

Fig. 1: CR performance Comparison

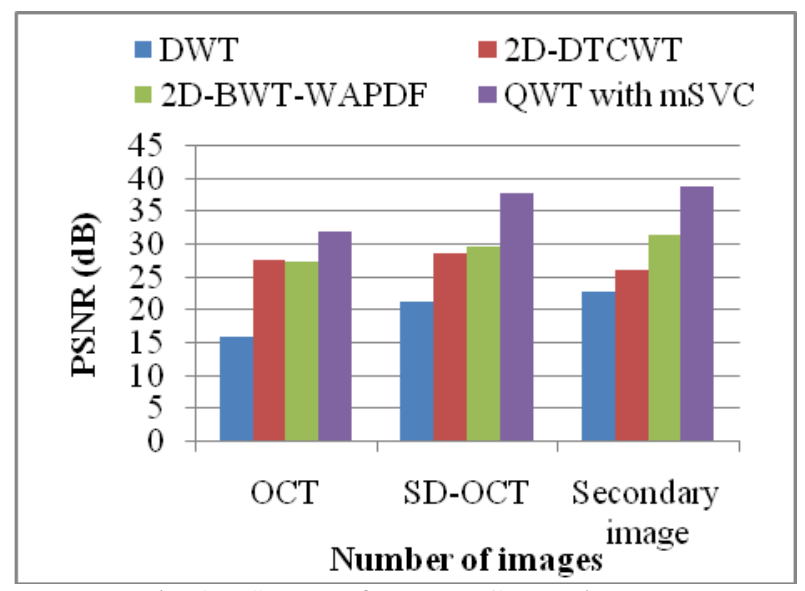

Fig. 2: PSNR Performance Comparison

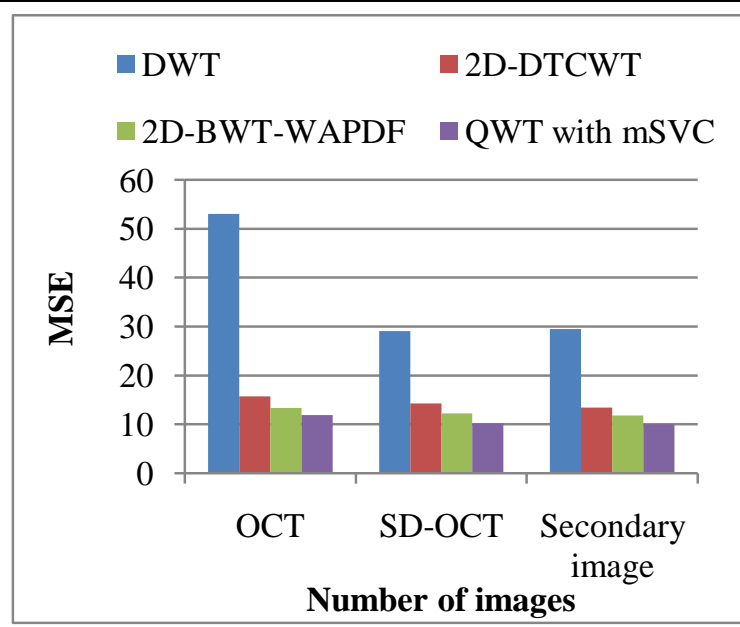

Fig. 3: MSE Performance Comparison

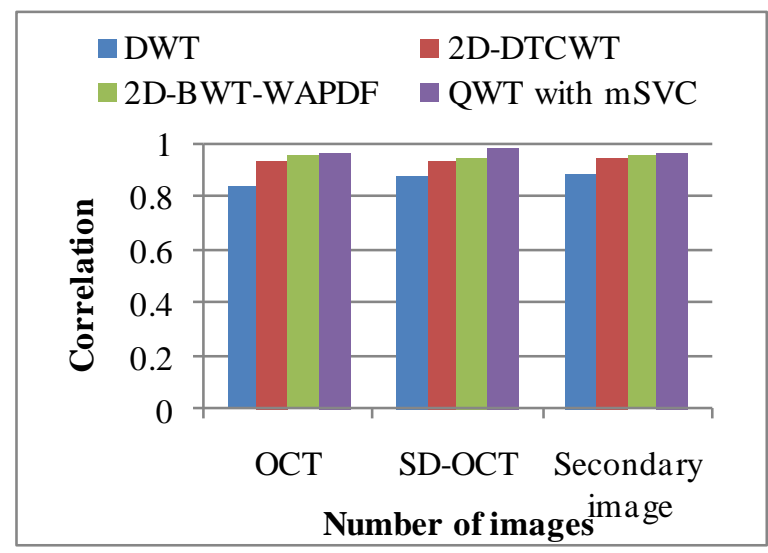

Fig. 4: Correlation Performance Comparison 


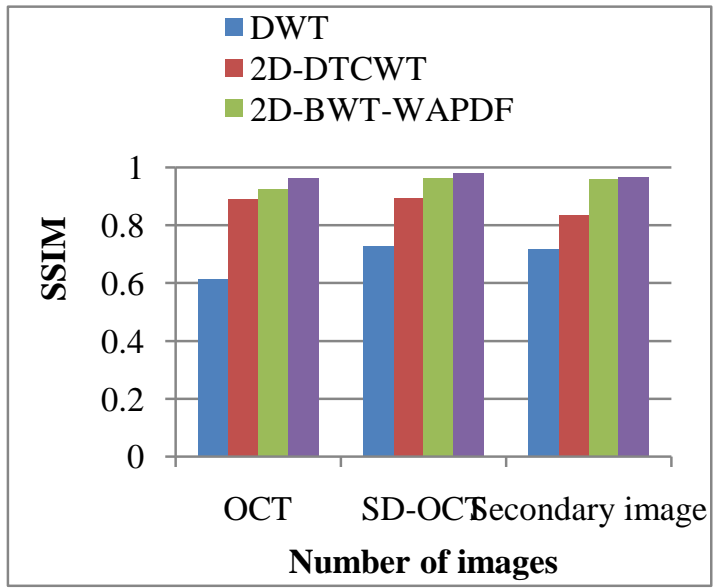

Fig. 5: SSIM Performance Comparison

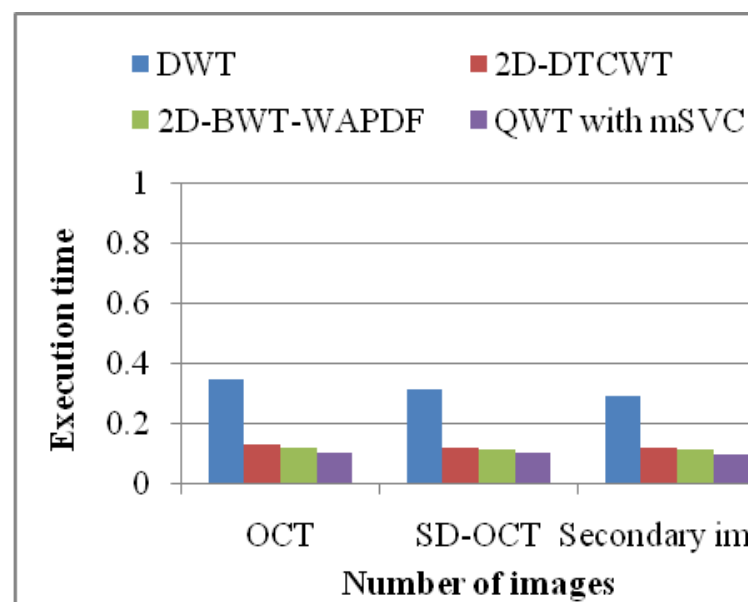

Fig. 6: Execution Time Performance Comparison

Fig. 1 shows that the CR performance comparison results between OCT, SD-OCT and secondary images using proposed QWT-mSVC, 2D- BWT with WAPDF, 2D-DTCWT and existing DWT. From the results, it is well known that proposed QWT - mSVC obtain high CR indicating the good purity of retrieved image. The main reason is that the clustering of large coefficients resembles that of the QWT; hence it concludes that QWT is a viable for SD-OCT images. The proposed QWT-mSVC method is more efficient for achieving higher $\mathrm{CR}$ values for all three images and it proves that the SD-OCT has higher CR than other images.

Fig. 2 shows that the PSNR comparison results between OCT, SD-OCT and secondary images using proposed QWT-mSVC, 2D- BWT with WAPDF, 2DDTCWT and existing DWT. From the results, it is well known that proposed QWT-mSVC obtain high PSNR indicating the good reconstructed image. The proposed scheme is based on a various principle which does not need any factorization of the dilation matrix and also more stable from a wavelet designer point of view. The proposed QWT-mSVC method is more efficient for achieving higher

www.ijcat.com
PSNR values for all three images and it proves that the secondary image has higher PSNR than other images.

Fig. 3 shows that the MSE performance comparison results between OCT, SD-OCT and secondary images using proposed QWT-mSVC, 2D- BWT with WAPDF, 2D-DTCWT and existing DWT. From the results, it is well known that proposed QWT-mSVC obtain less MSE indicating the good reconstructed image. The importance of proposed work lies in the likelihood of decreasing the rates for which the image quality remains acceptable. The proposed QWT-mSVC method is more efficient for achieving lower MSE values for all three images and it proves that the secondary image has lower MSE than other images.

Fig. 4 shows that the correlation comparison results between OCT, SD-OCT and secondary images using proposed QWT-mSVC, 2D- BWT with WAPDF, 2DDTCWT and existing DWT. From the results, it is well known that proposed QWT-mSVC obtain less MSE indicating the good reconstructed image. The proposed QWT with mSVC obtain better performance compare then existing methods. It is an effective way of getting rid of the less informative part of this redundancy without compromising perfect reconstruction. The proposed QWTmSVC method is more efficient for achieving higher correlation values for all three images and it proves that the SD-OCT has higher correlation than other images.

Fig. 5 shows that the SSIM performance comparison results between OCT, SD-OCT and secondary images using proposed QWT-mSVC, 2D- BWT with WAPDF, 2D-DTCWT and existing DWT. From the results, it is well known that proposed QWT with mSVC gidetain better SSIM compare then existing methods. The main reason is that the proposed quincunx transform has the least redundancy. The proposed QWT-mSVC method is more efficient for achieving higher SSIM values for all three images and it proves that the SD-OCT has higher SSIM than other images.

Fig. 6 shows that the execution time comparison results between OCT, SD-OCT and secondary images using proposed QWT-mSVC, 2D- BWT with WAPDF, 2D-DTCWT and existing DWT. From the results, it is well known that proposed QWT - mSVC obtain less execution time compare then existing methods. The proposed QWT$\mathrm{mSVC}$ method is more efficient for achieving lower time complexity values for all three images and it proves that the secondary image has fast execution time than other images.

\section{CONCLUSION}

In this research, the OCT, SD-OCT and secondary images are considered to analyze using proposed QWTmSVC, 2D-BWT with WAPDF, 2D-DTCWT and existing DWT methods. The DWT method provides lower PSNR, $\mathrm{CR}$, correlation, SSIM and higher MSE, execution time values. 2D-DTCWT method is used to compress the image efficiently and it provides experimental results better than the DWT method. The OCT, SD-OCT and secondary images are evaluated by using 2D-DTCWT method but it 
still has issue with lower quality in image compression results. To overcome the above mentioned issues, 2DBWT with WAPDF is used which helps to improve the image compression performance. This approach provides better performance metrics rather than the previous DWT and 2D-DTCWT methods. However it has problem along with time complexity and compression ratio performance. to avoid this problem, QWT with $\mathrm{mSVC}$ is introduced. It evaluates the OCT, SD-OCT and secondary images more effectively and provides higher PSNR, CR, correlation, SSIM and lower MSE, execution time values rather than the 2D-BWT with WAPDF, 2D-DTCWT and DWT methods. The experimental result proves that the proposed QWT with mSVC method has superior image compression performance than the other methods.

\section{REFERENCES}

1. S.S.Goh, Q.Jiang, and T.Xia, Construction of Biorthogonal Multiwavelets Using the Lifting Schemell, Applied and Computational Harmonic Analysis, pp-336- 352, 2000.

2. F.Keinert, Raising -Multiwavelet Approximation Order Through Lifting\|, SIAM Journal on Mathematical Analysis, pp1032-1049, 2001.

3. Singh, Anushikha, et al. "Image processing based automatic diagnosis of glaucoma using wavelet features of segmented optic disc from fundus image."Computer methods and programs in biomedicine 124 (2016): 108-120.

4. Monteiro, M. L. R., F. A. Medeiros, and M. R. Ostroscki. "Quantitative analysis of axonal loss in band atrophy of the optic nerve using scanning laser polarimetry." British journal of ophthalmology 87.1 (2003): 32-37.

5. Sanchez-Galeana, Cesar, et al. "Using optical imaging summary data to detect glaucoma." Ophthalmology 108.10 (2001): 18121818.

6. Zangwill, Linda M., et al. "Discriminating between normal and glaucomatous eyes using the Heidelberg retina tomograph, GDx nerve fiber analyzer, and optical coherence tomograph." Archives of ophthalmology 119.7 (2001): 985-993.

7. Subhasis Saha \& Rao Vemuri "Analysis based adaptive Wavelet filter selection in Lossy image coding schemes", ISCAS-2000-IEEE International Symposium on Circuits and systems May-28-31,2000, Genewa, Switzerland.

8. M. Hemalatha and S. Nithya, "Dual Tree Complex Wavelet Transform Based Compression of Optical Coherence Tomography Images for Glaucoma Detection using Modular Neural Network", Research Journal of Applied Sciences, Engineering and Technology 13(11): 825-834, 2016
9. R. J. Clarke, "Relation between the KarhunenLoeve and cosine transforms," IEE Proceedings, Part F: Communications, Radar and Signal Processing, vol. 128, no. 6, pp. 359-360, Nov. 1981.

10. V. Kunchigi, et al., "Simulation of Vedic Multiplier in DCT Applications," International Journal of Computer Applications, vol/issue: 63(16), pp. 27-32, 2013.

11. M. Beladgham, A. Bessaid, A. Moulay-Lakhdar, A. Taleb-Ahmed, Medical Image Compression Using Quincunx Wavelets and VQ Coding, International Review on Computers and Software (I.RE.CO.S.), Vol. 5, N. 6, November 2010

12. B. S. Harish, M. B. Revanasiddappa, S. V. Aruna Kumar, A Modifed Support Vector Clustering Method for Document Categorization, IEEE Interational Conference on Knowledge Engineering and Applications, 2016

13. V. Chappelier, Progressive coding of images by directed wavelet. Phd Thesis, Rennes1University, 2005. 